\title{
Efficacy of 1.5 Harmonic Imaging for Intravenous Myocardial Contrast Echocardiography
}

\author{
Tsutomu Toshida, MD, Fuminobu Ishikura, MD, Toshihiko Asanuma, MD, \\ Akiko Iwata, MD, Ayako Miki, MS, Kentaro Otani, MS and Shintaro Beppu, MD \\ Division of Functional Diagnostic Science, Graduate School of Medicine, Osaka University, \\ Suita, Japan
}

\begin{abstract}
Background. Although second harmonic $(2 \mathrm{H})$ imaging is utilized to minimize the tissue signals in myocardial contrast echocardiography (MCE), the native tissue harmonic signal is sufficiently high enough to cause a misreading of myocardial opacification when high mechanical index (MI) is used.

Purpose. The backscattered signal from microbubbles at high $\mathrm{Ml}$ is broadband, while it is narrowband at low Ml. Therefore, the bubble/tissue signal ratio is expected to be high between the fundamental signal and $2 \mathrm{H}$ frequencies at high MI. Our aim was to elucidate the efficacy of a new imaging method using an in-between frequency, denoted as 1.5 harmonic $(1.5 \mathrm{H})$ imaging.

Methods. Six open-chest dogs were examined using Toshiba APLIO with $1.5 \mathrm{H}$ and conventional $2 \mathrm{H}$ system. The transmitting/receiving frequencies were $2.3 / 3.3 \mathrm{MHz}$ for $1.5 \mathrm{H}$ and $1.4 / 2.8 \mathrm{MHz}$ for $2 \mathrm{H}$ imaging, respectively. Intermittent MCE was performed at a $\mathrm{MI}$ of 1.6 after injection of Levovist ${ }^{\circledR}$, and baseline intensity and peak intensity of opacification of the ventricular septum were measured.

Results. While baseline intensity of the septum was significantly lower using $1.5 \mathrm{H}$ than $2 \mathrm{H}(26 \pm 4$ vs. $43 \pm 14$ gray level, $\mathrm{p}<0.001)$, the opacification after Levovist was significantly higher using $1.5 \mathrm{H}$ than $2 \mathrm{H}(91 \pm 32 \mathrm{vs} .51 \pm 38$, $\mathrm{p}<0.001$ ). Due to the combination of these effects, visual recognition of myocardial opacification was quite superior with $1.5 \mathrm{H}$ imaging.

Conclusion. The $1.5 \mathrm{H}$ imaging system provides superb contrast opacification when using high Ml.
\end{abstract}

(J Echocardiogr 2005; 3: 104-108)

Key words: myocardial contrast echocardiography, harmonic, bubble

\section{Introduction}

Myocardial contrast echocardiography (MCE) is a superior method for detecting myocardial perfusion. Although intravenous MCE seems to be useful method for clinical setting, the backscatter from bubbles may not be strong enough to opacify the myocardium sufficiently because of as small size as passing through the

Received February 28, 2005; revision received October 2, 2005; accepted October 14, 2005

Address for correspondence: Fuminobu Ishikura, MD

Division of Functional Diagnostic Science, Graduate School of Medicine, Osaka University,

1-7 Yamadaoka, Suita 565-0871, Japan.

Telephone: +81-6-6879-2561

Fax: +81-6-6879-2561

E-mail: ishikura@sahs.med.osaka-u.ac.jp

(C) 2005 Japanese Society of Echocardiography pulmonary capillaries. To overcome this inferiority for imaging, many new techniques for enhancing bubble signals have being developed. They utilize the unique scattering behavior of microbubbles when they are exposed to ultrasound, such as second harmonic $(2 \mathrm{H})$ imaging [1, 2], intermittent imaging [3-5], pulse inversion imaging [6], sub-harmonic imaging [7], power modulation [8], power pulse-inversion imaging [9, 10], power Doppler imaging [11, 12] and ultraharmonic imaging [13]. By using these techniques, the echo signal of bubbles can be separated from that of tissues. However, it has been revealed that when the high acoustic power is used, some tissues reflect $2 \mathrm{H}$ frequency signals besides the fundamental one; this is called tissue harmonic [14]. It is conceivable that the tissue harmonic image overlaps the harmonic image from bubbles, resulting in some disturbance of visual 


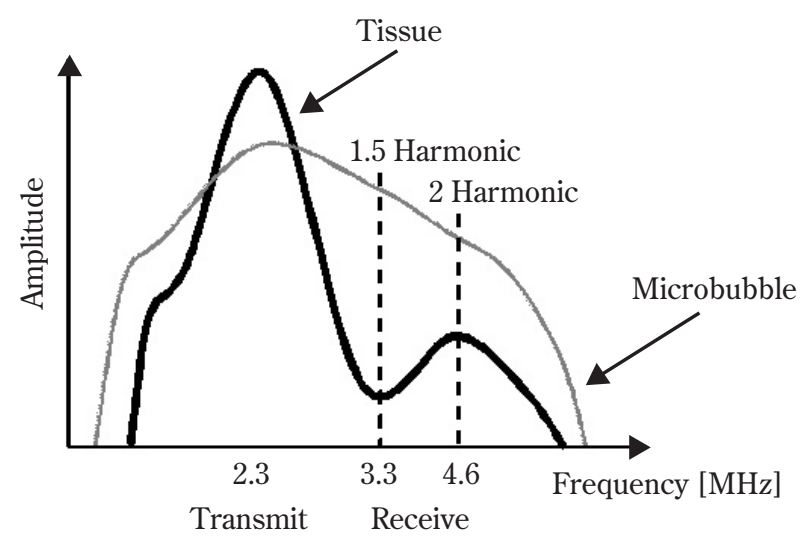

Fig. 1. The relationship between frequency and amplitude when the transmitting frequency was 2.3 $\mathrm{MHz}$ using a high mechanical index. The amplitude of backscatter signals from the microbubbles showed broadband distribution in the frequency domain, and the amplitude of backscatter signals from tissues showed two peaks at the fundamental and 2 harmonic frequency. The difference in amplitude between microbubbles and tissues was prominent at 3.3 $\mathrm{MHz}$ frequency, which was the midpoint between the fundamental and 2 harmonic frequency.

At this frequency, which was denoted 1.5 harmonic, the bubble signals were clearly separated from the tissue signals.

assessment of MCE [15].

It is known that microbubbles are not oscillated but destructed by high acoustic power insonation, followed by radiation of broadband ultrasound [16]. This frequency range covers from fundamental to over $2 \mathrm{H}$ frequency. Tissue signal, however, is low within this frequency domain. Therefore, the bubble/tissue signal ratio ( $\mathrm{B} / \mathrm{T}$ ratio) becomes high, when the receiver frequency is set between fundamental and $2 \mathrm{H}$ (Figure 1). This is the characteristic concept of the 1.5 harmonic $(1.5 \mathrm{H})$ imaging of MCE.

Our aim was to elucidate the efficacy of $1.5 \mathrm{H}$ imaging for myocardial opacification with the triggered intermittent mode at high mechanical index (MI) in an animal study.

\section{Methods}

\section{Animal Preparation}

Six healthy dogs weighing 12.5 to $14.0 \mathrm{~kg}$ (mean \pm $\mathrm{SD}, 13.7 \pm 0.6 \mathrm{~kg}$ ) were used to assess the MCE. This study was approved by the Osaka University Medical
School Animal Care and Use Committee and was in compliance with the Osaka University Medical School guidelines for the care and use of laboratory animals. Dogs were anesthetized with intravenous pentobarbital sodium $(35 \mathrm{mg} / \mathrm{kg}$ ), and continuous anesthesia (6 to 8 $\mathrm{mg} / \mathrm{kg} /$ hour) was maintained during the experiment. The dogs were intubated with a cuffed endotracheal tube and ventilated by room air with a Harvard-type respirator (Model SN-480-3, Shinano Manufacturing Co Ltd, Tokyo, Japan). Left lateral thoracotomy was performed, and the heart was suspended in a pericardial cradle. The animals received saline at approximately $1 \mathrm{ml} / \mathrm{min}$ during the surgical procedure.

\section{Myocardial Contrast Echocardiography}

Left ventricular short axis images were obtained in open-chest dogs using a Toshiba SSA-770A instrument (APLIO), (Tokyo, Japan), equipped with conventional $2 \mathrm{H}$ imaging and newly developed $1.5 \mathrm{H}$ imaging system. The transducer (25AT) was placed on the myocardium via an acoustic sheet (Sonar-AID, Geistlich-Pharma, Switzerland) and fixed into position with a hand-made clamp to obtain a short axis view of the heart.

The transmit acoustic power was fixed at a MI of 1.6. The dynamic range was set at $65 \mathrm{~dB}$, and the ultrasound focus was set at the upper third $(3 \mathrm{~cm})$ of the imaging field. Other instrument settings, such as gain, compensation, and depth at baseline were optimized for each study but kept constant throughout the study.

The contrast agent for MCE was Levovist ${ }^{\circledR}$ (Schering AG, Berlin, Germany), which contains microbubbles composed of air and palmitin acid shell. The Levovist solution $(300 \mathrm{mg} / \mathrm{ml})$ was reconstituted by the addition of $6.8 \mathrm{ml}$ of sterile water, and $0.5 \mathrm{ml}$ of Levovist was administered by a slow bolus injection (approximately $1 \mathrm{ml} /$ second) through a thin tube inserted into a peripheral vein, followed by a saline flush.

At $1.5 \mathrm{H}$ imaging, the transmitting/receiving frequency was $2.3 / 3.3 \mathrm{MHz}$ with pulse subtraction mode. For conventional $2 \mathrm{H}$ imaging, the same transducer was used with transmitting/receiving frequency of 1.4/2.8 $\mathrm{MHz}$. MCE images were obtained in intermittent mode at every 4 cardiac cycles, in which the end-systolic phase was triggered, and was recorded on S-VHS videotape. The experiments for each harmonic imaging were repeated twice, and a total of 4 injections were performed. The interval of each injection was set long enough until the effect of the previous injection 
disappeared.

\section{MCE Image Analysis}

The video intensity (VI) of the septum was analyzed by gray scale of 256 levels using an off-line image analysis system (Color Cardiology Work Station, TomTec, USA). A round region of interest (ROI) of 1.0 $\mathrm{cm}$ in diameter was set at the ventricular septum, and the VI was measured in 3 consecutive end-systolic frames, and the average was calculated before and after contrast injection. The increment in VI was calculated by subtracting the baseline intensity from peak intensity on the MCE image. The average value by the two injections of the contrast agent was adopted for each dog.

\section{Statistical Analysis}

Data is expressed as average \pm standard deviation (SD). Comparison of the average of each group was performed by the paired t-test. A p-value less than 0.05 was considered statistically significant.

\section{Results}

\section{Baseline Images}

Before administration of Levovist, the VI of the myocardium was low using $1.5 \mathrm{H}$ imaging (Figure $2 \mathrm{C}$ ), while it was heterogeneously high using $2 \mathrm{H}$ imaging (Figure 2A). The baseline VI of the ventricular septum was significantly lower using $1.5 \mathrm{H}$ imaging than using $2 \mathrm{H}$ imaging (26 \pm 4 vs. $43 \pm 14, \mathrm{p}<0.001$ ) (Figure 3 ).

\section{Myocardial Opacification}

After administration of Levovist, the myocardium was adequately opacified using both $1.5 \mathrm{H}$ and $2 \mathrm{H}$ imaging (Figure 2, B and D). The VI of the opacified septum was significantly higher using $1.5 \mathrm{H}$ imaging than $2 \mathrm{H}$ imaging $(116 \pm 31$ vs. $94 \pm 29, \mathrm{p}<0.001)$, and
Baseline
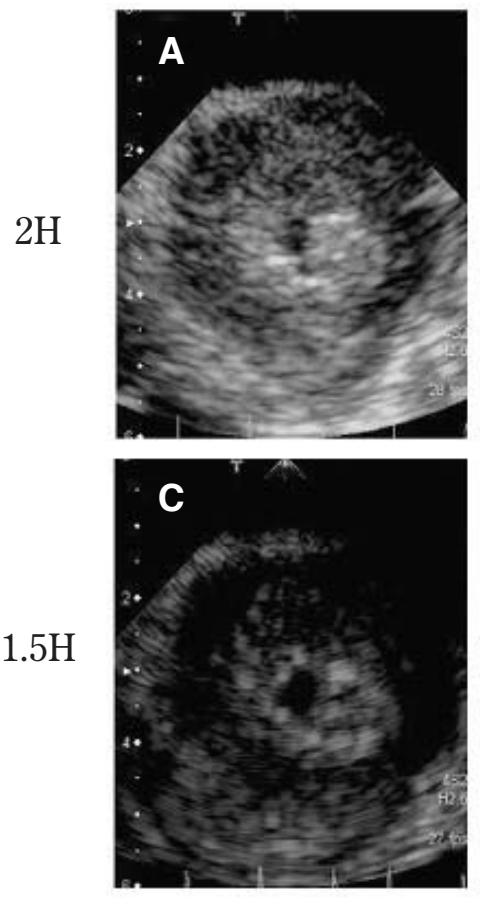

Contrast
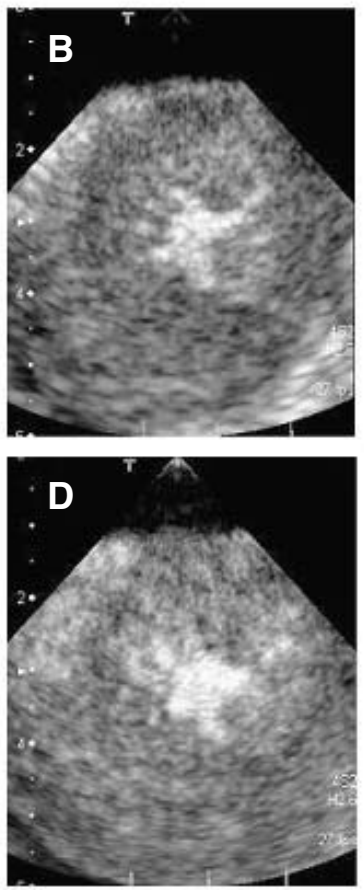

Fig. 2. The left ventricular short axis view of 2 harmonic $(2 \mathrm{H})$ imaging (upper panels) and 1.5 harmonic $(1.5 \mathrm{H})$ imaging (lower panels) before and after injection of Levovist.

The myocardial intensity of $1.5 \mathrm{H}$ imaging at baseline was lower than that of $2 \mathrm{H}$ imaging, as shown in the left panels. On the other hand, the opacification of myocardium using $1.5 \mathrm{H}$ imaging after injection of Levovist, was higher than that using $2 \mathrm{H}$ imaging, as shown in the right panels.

In consequence, the visual recognition of the myocardial opacification using $1.5 \mathrm{H}$ imaging was more reliable than that using $2 \mathrm{H}$ imaging. 
Video intensity

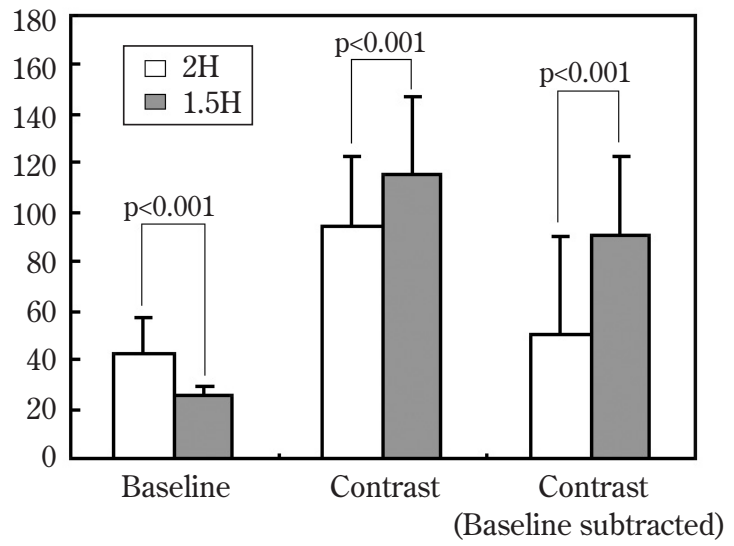

Fig. 3. The video intensity of myocardium using 2 and 1.5 harmonic imaging before and after injection of Levovist.

The video intensity of the septal segments using 2 harmonic imaging at baseline was significantly higher at $43 \pm 14$ than that using 1.5 harmonic imaging $(26 \pm 4)$. $(p<0.001)$.

The video intensity of the septal segments using 2 harmonic imaging increased to $94 \pm 29$ after injection of Levovist, which was significantly lower than that using 1.5 harmonic imaging, which increased to $116 \pm 31 .(p<0.001)$.

The increment in VI from the baseline value was significantly higher using 1.5 harmonic imaging than 2 harmonic imaging $(91 \pm 32$ vs. $51 \pm 38, p<0.001$ )

the increment in VI from the baseline value was significantly higher using $1.5 \mathrm{H}$ imaging than $2 \mathrm{H}$ imaging (91 \pm 32 vs. $51 \pm 38, \mathrm{p}<0.001$ ) (Figure 3 ).

\section{Discussion}

Intermittent mode is the effective technique for myocardial opacification of the microbubbles via intravenous injection [3]. The $2 \mathrm{H}$ imaging is also superb technology for enhancing the backscatter from microbubbles. Initially, the $2 \mathrm{H}$ technique had been developed to differentiate echo of bubbles from that of tissues. Therefore, the combination of intermittent mode and $2 \mathrm{H}$ mode is used widely for assessing myocardial perfusion abnormalities. However, the inevitable adverse effect of using high acoustic power for the intermittent mode is the generation of tissue harmonic signals. Tissue harmonic imaging itself is actually a useful technique for eliminating noise signals creating a clear ultrasound image [14]. At MCE, however, it overlaps the $2 \mathrm{H}$ image of bubbles, resulting in interference of the visual assessment of myocardial opacification [15].

Therefore, the idea of using a frequency between the fundamental and $2 \mathrm{H}$ frequencies seemed to be smart in order to eliminate the tissue harmonic signals. As shown in the present study, the $1.5 \mathrm{H}$ imaging is quite useful for increasing the $\mathrm{B} / \mathrm{T}$ ratio by decreasing the baseline intensity of the tissue and increasing the bubble intensity.

Using conventional $2 \mathrm{H}$ imaging for $\mathrm{MCE}$, baseline intensity of the myocardium should be subtracted from the opacified myocardial intensity to evaluate myocardial perfusion. The problem is the validity of subtracting the intensity because the VI is the logarithmic compression of acoustic power. Subtraction of the logarithm means a ratio of raw value. We need to convert the video intensity or decibel value into the raw value, such as acoustic power, to evaluate the myocardial opacification quantitatively, which is somewhat time consuming. Therefore, to avoid the complicated mathematics and to evaluate the opacification correctly, the baseline intensity of the myocardium should be null or extremely low. It is natural that visual recognition of the myocardial opacification should be easy when the baseline intensity of the myocardium is null. In this sense, $1.5 \mathrm{H}$ imaging is the convenient method for MCE.

Reduction of the baseline intensity of the myocardium may have unexpected problems. The cross sectional plane during MCE should be adjusted properly so it dose not move or tilt at the clinical setting. However, because of a faint echo of the ventricular wall and valves, adequate adjustment of the cross-sectional plane of echocardiography may be difficult. Another possible problem is the gain setting. Usually the gain can be set adequately by looking at the monitor intensity of the ventricular wall and valves. At $1.5 \mathrm{H}$ imaging, however, gain control may not work definitely at the baseline stage because of the extremely low intensity. When the gain setting at the baseline state is high, the intensity of bubbles may be excessive for evaluation of opacification. In the present study, we set the gain optimally at the conventional fundamental mode and did not change it in any harmonic mode during the experiment. It should be examined at the optimal gain setting for the $1.5 \mathrm{H}$ study.

\section{Study Limitations}

We applied triggering intervals at every 4 cardiac 
cycles and did not evaluate the effect of another triggering interval on regional video intensities. Indices of myocardial perfusion are calculated from the replenishment curve, which is made by changing the triggering interval. We examined only the opacification effect by $1.5 \mathrm{H}$ and did not examine the influence from the triggering interval. We speculate that the shape or the measured value of replenishment curve is not different between conventional $2 \mathrm{H}$ and newly developed $1.5 \mathrm{H}$, but do not have any data.

The magnitude of harmonic backscatter depends on the transmitting acoustic power. We consider that this is also applied to $1.5 \mathrm{H}$ imaging. In the present study, however, we did not examine the effect of MI. We studied the effect of $1.5 \mathrm{H}$ at maximal MI of the equipment: MI of 1.6.

The method of administration of contrast agent, Levovist, may be criticized. Slow bolus injection was used in this experiment. In general, continuous administration method is utilized to maintain the constant density of contrast agent. This is essential for the evaluation of efficacy of MCE. In the present study, however, we had to evaluate the baseline intensity before contrast in each harmonic imaging. Despite slow bolus injection, we had to wait some time until the contrast effect was absorbed. We intended to inject the contrast agent steadily and constantly during the experiment. Nevertheless, there should be some variety of injection speed and amount.

\section{Conclusion}

The $1.5 \mathrm{H}$ imaging minimizes the signal from tissue and enhances the signal from bubbles, which is a superb property for MCE.

\section{References}

1. Crouse LJ, Kramer PH. Second Harmonic imaging: Good reverberations. Am Heart J 1999; 138: 19-20.

2. Kornbluth M, Liang DH, Paloma A, Schnittger I. Native tissue harmonic imaging improves endocardial border definition and visualization of cardiac structures. J Am Soc Echocardiogr 1998; 11: 693-701.

3. Porter TR, Xie F, Li S, D'Sa A, Rafter P. Increased ultrasound contrast and decreased microbubble destruction rates with triggered ultrasound imaging. J Am Soc Echocardiogr 1996; 9: 599-605.

4. Kamiyama N, Moriyasu F, Mine Y, Goto Y. Analysis of flash echo from contrast agent for designing optimal ultrasound diagnostic systems. Ultrasound Med Biol
1999; 25: 411-420.

5. Frinking PJ, Bouakaz A, Kirkhorn J, Ten Cate FJ, de Jong $\mathrm{N}$. Ultrasound contrast imaging: current and new potential methods. Ultrasound Med Biol 2000; 26: 965-975.

6. Burns PN, Hope SD, Averkiou MA. Nonlinear imaging. Ultrasound Med Biol 2000; 26 (Suppl 1): S19-22.

7. Shankar PM, Dala Krishna P, Newhouse VL. Advantages of subharmonic over second harmonic backscatter for contrast-to-tissue echo enhancement. Ultrasound Med Biol 1998; 24: 395-399.

8. Caiani EG, Lang RM, DeCara J, et al. Objective assessment of left ventricular wall motion from contrastenhanced power modulation images. J Am Soc Echocardiogr 2002; 15: 118-128.

9. Tiemann K, Veltmann C, Ghanem A, et al. The impact of emission power on the destruction of echo contrast agents and on the origin of tissue harmonic signals using power pulse-inversion imaging. Ultrasound Med Biol 2001; 27: 1525-1533.

10. Tiemann K, Lohmeier S, Kuntz S. Real-time contrast echo assessment of myocardial perfusion at low emission power: first experimental and clinical results using power pulse inversion imaging. Echocardiography 1999; 16: 799809 .

11. Becher H, Tiemann K, Schlief R, Luderitz B, Nanda NC. Harmonic power doppler contrast echocardiography; Preliminary clinical results. Echocardiography 1997; 14: 637-642.

12. Hirooka K, Miyatake K, Hanatani A, et al. Enhanced methods for visualizing myocardial perfusion with peripheral venous injection of Levovist: application of triggered harmonic imaging and triggered harmonic power Doppler imaging techniques. Int J Card Imaging 2000; 16 : 233-246.

13. Kuersten B, Murthy TH, Li P, et al. Ultraharmonic myocardial contrast imaging: in vivo experimental and clinical data from a novel technique. J Am Soc Echocardiogr 2001; 14: 910-916.

14. Thomas JD, Rubin DN. Tissue harmonic imaging: why dose it work? J Am Soc Echocardiogr 1998; 11: 803-808.

15. Pelberg RA, Wei K, Kamiyama N, Sklenar J, Bin J, Kaul S. Potential advantage of flash echocardiography for digital subtraction of B-mode images acquired during myocardial contrast echocardiography. J Am Soc Echocardiogr 1999; 12: 85-93.

16. Porter TR, Xie F. Transient myocardial contrast after initial exposure to diagnostic ultrasound pressures with minute doses of intravenously injected microbubles. Demonstration and potential mechanism. Circulation 1995; 92: 2391-2395. 